\title{
Fixed point and rigidity theorems for harmonic maps into NPC spaces
}

\author{
Georgios Daskalopoulos ${ }^{1}$ \\ Brown University \\ daskal@math. brown.edu \\ Chikako Mese $^{2}$ \\ Johns Hopkins University \\ cmese@math.jhu.edu
}

\begin{abstract}
We show that harmonic maps from 2-dimensional Euclidean polyhedra to arbitrary NPC spaces are totally geodesic or constant depending on a geometric and combinatorial condition of the links of the 0-dimensional skeleton. Our method is based on a monotonicity formula rather than a codimension estimate of the singular set as developed by Gromov-Schoen or the mollification technique of Korevaar-Schoen.
\end{abstract}

\section{Introduction}

The connection between harmonic maps and representations of discrete groups has been studied extensively in recent years. A harmonic map is classically defined between Riemannian manifolds and is a critical point of the energy functional. With the seminal work of Gromov and Schoen on $p$-adic superrigidity (cf. [GS] and also [KS1], [KS2]), one can employ techniques from geometric analysis to study harmonic maps from Riemannian manifolds to metric spaces of non-positive curvature (NPC spaces). Under appropriate curvature assumptions, one can prove that the harmonic map is totally geodesic or even constant and conclude this way that it is rigid. In the case the harmonic map is equivariant with respect to a representation of the fundamental group of the domain manifold to the isometry group of an NPC space, one can deduce the rigidity of the representation. Instead of smooth domains, one could also ask about the rigidity of representations of the fundamental group of a singular space like a piecewise smooth polyhedron into the isometry group of an NPC space. These questions are motivated by the study

\footnotetext{
${ }^{1}$ supported by research grant NSF DMS-0604930

${ }^{2}$ supported by research grant NSF DMS-0450083
} 
of representations of lattices in non-Archimedian groups in connection with Margulis superrigidity.

Harmonic maps from singular domains were first studied in [Ch] and further elaborated in [EF], [DM1] and [DM2]. More precisely, let $X$ be an admissible, Euclidean, 2-dimensional simplicial complex (cf. Section 2 for precise definitions). We will denote by $X^{(i)}$ the $i$ th skeleton of $X, \tilde{X}$ its universal cover and $\Gamma=\pi_{1}(X)$. Let $Y$ be an NPC space and let $\rho: \Gamma \rightarrow \operatorname{Isom}(Y)$ be a representation of $\Gamma$ via isometries on $Y$. Under very general assumptions on $\Gamma$ and $\rho$, one can construct a $\Gamma$-equivariant energy minimizer $f: \tilde{X} \rightarrow Y$, also called a harmonic map (cf. [DM1] for details). For reasons explained in [DM2], it is useful to associate certain weights $w$ to the 2 -skeleton of $X$ and modify the notion of energy to account for the weights. One then obtains the notion of a $w$-harmonic map (cf. Section 2 for details).

Perhaps the most interesting feature of harmonic (or $w$-harmonic) maps from singular domains is that they are Hölder continuous, but in general fail to be Lipschitz continuous. Given $p \in X$, let $\operatorname{ord}(p)$ denote the order of $f$ at $p$. As in the case of smooth domains, $\operatorname{ord}(p)$ can be identified with the degree of homogeneity of the blow-up map of $f$ and the Hölder constant of $f$ (cf. [DM1] and Section 2). If $\operatorname{ord}(p) \geq 1$ for all $p$, then the map is Lipschitz continuous and combined with a Bochner formula, one can obtain rigidity of $f$; in other words that the $w$-harmonic map $f$ is totally geodesic on each simplex of $X$ or even a constant map. In the case of a smooth target $Y$, this was one of the main results of [DM2].

The goal of the present paper is to extend these results in the following ways. First, we consider maps to arbitrary NPC space targets. These include important examples like trees or the Weil-Petersson completion of Teichmüller space. Moreover, we allow our domain space to be polyhedra with arbitrary Euclidean metrics rather polyhedra consisting of simplices isometric to equilateral triangles. This makes our theorems applicable to a larger variety of examples than considered in [DM2] and the combinatorial approach of [W1], [W2] and [IN] discussed below. The main results of the paper can be summarized as follows:

Theorem [cf. Theorem 13] Let $X$ be a 2-dimensional admissible Eucliean simplicial complex, $Y$ an NPC space and $f: \tilde{X} \rightarrow Y$ a $\Gamma$ equivariant $w$-harmonic map such that $\operatorname{ord}(p) \geq 1$ for all $p \in X^{(0)}$. Then $f$ is totally geodesic on each 2-simplex $F$ of $X$. If the curvature of $Y$ is strictly negative, then $f$ maps each 2-simplex $F$ into a geodesic. 
If $\operatorname{ord}(p)>1$ for all $p \in X^{(0)}$, then $f$ is constant.

As for smoth targets, the order of a harmonic map at a 0 -simplex $p$ is related to certain geometric and combinatorial information of $X$. Let $\lambda_{1}\left(\operatorname{Lk}(p), T_{Q} Y\right)$ denote the first eigenvalue of $\operatorname{Lk}(p)$, the link of $p$ in $X$ with values in the tangent cone $T_{Q} Y$ of $Y$ (cf. Sections 2 and 4 ) for details. We show:

Theorem [cf. Theorem 16] Let $X$ and $Y$ be as in above and $f: \tilde{X} \rightarrow Y$ a $\Gamma$-equivariant $w$-harmonic map. If $\lambda_{1}\left(L k(p), T_{Q} Y\right) \geq \beta(>\beta)$ for all $Q \in Y$, then $\operatorname{ord}(p) \geq \sqrt{\beta}(>\sqrt{\beta})$.

By combining the previous two theorems, we obtain:

Theorem [cf. Theorem 17] Suppose $\lambda_{1}\left(L k(p), T_{Q} Y\right) \geq 1$ for all $p \in X^{(0)}$ and all $Q \in Y$. Then any $w$-harmonic map $f: \tilde{X} \rightarrow Y$ is totally geodesic on each simplex of $X$. If $Y$ is strictly negatively curved, then $f$ maps each simplex of $X$ into a geodesic. Furthermore, if $\lambda_{1}\left(L k(p), T_{Q} Y\right)>1$ for all $p \in X^{(0)}$ and all $Q \in Y$, then $f$ is constant.

We now give a sketch of the proofs of the above theorems. Let $f: \tilde{X} \rightarrow$ $Y$ be a $\Gamma$-equivariant $w$-harmonic map as before. As mentioned above, the condition $\operatorname{ord}(p) \geq 1$ for all $p \in X^{(0)}$ implies that $f$ is Lipschitz and this is a key part of the argument. Next, by performing domain variations along the $\mathrm{x}$-axis we obtain as in [KS1], the subharmonicity of $\left|\frac{\partial f}{\partial x}\right|^{2}$ (cf. Lemma 5). Since variations in the $y$-direction do not make sense along the edges, we have to appeal to the balancing condition and the holomorphicity of the Hopf differential to obtain the subharmonicity of $\sum_{F \in \mathcal{F}(E)}\left|\frac{\partial f_{F}}{\partial y}\right|^{2}$, where the sum is taken over all the faces of $\mathrm{X}$ adjacent to the edge $E$ (cf. Lemma 5). This implies the subharmonicity of the energy density $|\nabla f|^{2}$ (cf. Lemma 6). In particular, the energy density $|\nabla f|^{2}$ is of Sobolev class $W^{1,2}$ and hence it has sufficient regularity to justify our main monotonicity formula (cf. Lemmas 9, 10 and 11). The weak inequality $\triangle|\nabla f|^{2} \geq 0$ defined on each face of $X$ is now summed over all the faces of $X$. In [DM2], we used Stokes' Theorem on each face and balancing condition along the boundary of each face to justify the conclusion that this sum is zero which immediately implies the harmonicity of $|\nabla f|$. Because of the singular nature of the target 
space considered in this paper, we use an alternate argument based on the monotonicity formula (cf. Theorem 12) to deduce a more general conclusion. This implies that the pullback tensor

$$
\left(\pi_{i j}\right)=\left(\begin{array}{cc}
\left|\frac{\partial f}{\partial x}\right|^{2} & \frac{\partial f}{\partial x} \cdot \frac{\partial f}{\partial y} \\
\frac{\partial f}{\partial x} \cdot \frac{\partial f}{\partial y} & \left|\frac{\partial f}{\partial y}\right|^{2}
\end{array}\right)
$$

is smooth on each simplex. The NPC condition on the target space implies that this pullback tensor $\pi_{i j}$ is also NPC (cf. Appendix). This, combined with the harmonicity of its components, implies that $\pi_{i j}$, and hence also the map $f$, is flat (cf. Theorem 13). The totally geodesic property or the constancy of the map $f$ follows.

We next indicate how to relate the order of $f$ at $p$ with the eigenvalues of the link at $p$. In the case when the target $Y$ is smooth, this can be done by reducing the harmonic map equation of the blow up map to the eigenvalue equation of the Laplacian on $L k(p)$ (cf.[DM2]). In the case $Y$ is singular, we adopt the Rayleigh quotient definition of eigenvalue and relate it to the order (cf. Theorem 16). At this point our approach is parellel to the combinatorial approach of M-T. Wang and Izeki and Nayatani (cf. [W1], [W2] and [IN]). We note that for $X$, a 2-complex with a property that each 2 -simplex is isometric to an equilateral triangle, and a smooth target $Y$ we showed in [DM2] an explicit relation between the condition $\lambda_{1}(\operatorname{Lk}(p)) \geq 1$ and the corresponding condition for the eigenvalue of the combinatorial Laplacian $\lambda_{1}^{\operatorname{comb}}(\operatorname{Lk}(p)) \geq 1 / 2$.

We end this introduction by mentioning one important application of our results to rigidity questions of the mapping class group. Recently Wolpert showed that the tangent cones of the Weil-Petersson completion $\overline{\mathcal{T}}$ of the Teichmüller space $\mathcal{T}$ of marked genus g, n-punctured Riemann surfaces are Euclidian cones. In this case, the condition

$$
\lambda_{1}\left(\operatorname{Lk}(p), T_{Q} \overline{\mathcal{T}}\right) \geq 1
$$

can be replaced by the simpler $\lambda_{1}(\operatorname{Lk}(p)) \geq 1$. Therefore, we immediately obtain the following rigidity result:

Theorem [cf. Theorem 18] Let $X$ be a 2-dimensional admissible simplicial complex such that $\lambda_{1}(L k(p)) \geq 1$ for all $p \in X^{(0)}$. Then any $w$ harmonic map to the Weil-Petersson completion $\overline{\mathcal{T}}$ of Teichmüller space is totally geodesic on each simplex of $X$. Furthermore, if $\lambda_{1}(L k(p))>1$ for all $p \in X^{(0)}$ then $f$ is constant. 
The previous theorem clearly implies that given a group $\Gamma$ which is the fundamental group of a 2-complex $X$ admitting an Euclidean metric with the property that $\pi_{1}(X)=\Gamma$ and $\lambda_{1}(\operatorname{Lk}(p))>1$ for all $p \in$ $X^{(0)}$, then any representation of $\Gamma$ into the mapping class group is constant. In the special case $\Gamma$ is realized as a fundamental group of a 2-complex $X$ where all simplices are equilateral triangles with the condition $\lambda_{1}(\operatorname{Lk}(p))>1$ for all $p \in X^{(0)}$, this result was obtained by using discrete harmonic maps (cf. [IN] and [Wo]). In this case, the condition $\left.\lambda_{1}(\operatorname{Lk}(p))>1\right)$ is replaced by the combinatorial condition $\lambda_{1}^{c o m b}(\operatorname{Lk}(p))>1 / 2$, which are shown to be equivalent by Proposition 13 and Corollary 14 of [DM2]. Although technically simpler than ours, the Bochner formula for discrete harmonic maps does not seem to carry over to the case $\lambda_{1}^{\text {comb }}(\operatorname{Lk}(p))=1 / 2$ or for the apparantly more general condition that we are considering for $\Gamma$. As pointed out by M-T. Wang (cf. [W2]), there are important examples of complexes where the combinatorial eigenvalue of the Laplacian is equal to $1 / 2$.

Acknowledgement. We would like to thank Richard Wentworth for pointing out Wolpert's paper [Wo] to us.

\section{Definitions and known results}

\subsection{Admissible Euclidean complexes}

A simplicial complex of dimension 2 is referred to as a 2-complex. A connected locally finite 2-complex is called admissible (cf. [Ch] and $[\mathrm{EF}])$ if the following two conditions hold:

(i) $X$ is dimensionally homogeneous, i.e., every simplex is contained in a 2-simplex, and

(ii) $X$ is locally 1 -chainable, i.e., for any 0 -simplex $v$, every two 2 simplices $A$ and $B$ containing $v$ can be joined by a sequence $A=$ $F_{0}, e_{0}, F_{1}, e_{1}, \ldots, F_{k-1}, e_{k-1}, F_{k}=B$ where $F_{i}$ is a 2 -simplex containing $v$ and $e_{i}$ is a 1-simplex contained in $F_{i}$ and $F_{i+1}$.

The boundary $\partial X$ of $X$ is the union of 1-simplices that are contained in only one 2-simplex. A Riemannian 2-complex is a 2-complex $X$ along with a Riemannian metric $g_{F}$ defined on each 2-simplex $F$ smooth up to the boundary of $F$ so that for any two 2-simplices $F$ and $F^{\prime}$ sharing 
a 1-simplex $e, g_{F}$ and $g_{F^{\prime}}$ induce the Riemannian metric on $e$. We call a Riemannian 2-complex Euclidean if for any 2 -simplex $F$, there exists a simpicial isometry $\phi_{F}:\left(F, g_{F}\right) \rightarrow T$ where $T$ is a triangle in $\mathbf{R}^{2}$ with the induced Euclidean metric. In the sequel, all complexes are admissible, Euclidean, compact and without boundary. We will assume all simplices are closed and use $X^{(i)}$ to denote the $i$-skeleton of $X$, i.e. the union of all $i$-simplices of $X$. For any simplex $s, \operatorname{star}(s)$ will denote the union of all simplices containing $s$.

\section{$2.2 \quad$ NPC spaces}

A complete metric space $(Y, d)$ is called an NPC space if the following conditions are satisfied:

(i) The space $(Y, d)$ is a length space. That is, for any two points $P$ and $Q$ in $Y$, there exists a rectifiable curve $c$ so that the length of $c$ is equal to $d(P, Q)$ (which we will sometimes denote by $d_{P Q}$ for simplicity). We call such distance realizing curves geodesics.

(ii) For any three points $P, R, Q \in Y$, let $c:[0, l] \rightarrow Y$ be the arclength parameterized geodesic from $Q$ to $R$ and let $Q_{t}=c(t l)$. Then

$$
d_{P Q t}^{2} \leq(1-t) d_{P Q}^{2}+t d_{P R}^{2}-t(1-t) d_{Q R}^{2} .
$$

We say that the curvature of $(Y, d)$ is strictly negative if there exists $\kappa<0$ so that

$$
\begin{aligned}
& \cosh \left(-\kappa d_{P Q_{t}}\right) \\
& \leq \frac{\sinh \left(-(1-t) \kappa d_{Q R}\right)}{\sinh \left(-\kappa d_{Q R}\right)} \cosh \left(-\kappa d_{P Q}\right)+\frac{\sinh \left(-\kappa t d_{Q R}\right)}{\sinh \left(-\kappa d_{Q R}\right)} \cosh \left(-\kappa d_{P R}\right) .
\end{aligned}
$$

We now recall the notion of a tangent cone of an NPC space $Y$ at a point $Q \in Y$. Let $G_{Q} Y$ be a set of nonconstant arclength parameterized geodesics $c$ so that $c(0)=Q$. Given $c, c^{\prime} \in G_{Q} Y$, define

$$
\bar{L}\left(c(t), c^{\prime}\left(t^{\prime}\right)\right)=\cos ^{-1}\left(\frac{d_{Q c(t)}^{2}+d_{Q c^{\prime}\left(t^{\prime}\right)}^{2}-d_{c(t) c^{\prime}\left(t^{\prime}\right)}^{2}}{2 d_{Q c(t)} d_{Q c^{\prime}\left(t^{\prime}\right)}}\right)
$$

and

$$
\angle\left(c, c^{\prime}\right)=\lim _{t, t^{\prime} \rightarrow 0} \bar{Z}\left(c(t), c^{\prime}\left(t^{\prime}\right)\right) .
$$


Define an equivalence relation $c \approx c^{\prime}$ iff $\angle\left(c, c^{\prime}\right)=0$. The completion of the quotient $S_{Q} Y=\left(G_{Q} Y / \approx\right)$ with the distance function induced by $\angle$ is called the space of directions at $Q$. The tangent cone is the cone over $S_{Q} Y$, namely

$$
T_{Q} Y=\left(S_{Q} Y \times \mathbf{R}^{+}\right) /\left(S_{Q} Y \times\{0\}\right) .
$$

For $W=(V, t), W^{\prime}=\left(V^{\prime}, t^{\prime}\right) \in T_{Q} Y$, the distance function $d_{T_{Q} Y}$ is defined by

$$
d_{T_{Q} Y}^{2}=\left(W, W^{\prime}\right)=t^{2}+t^{\prime 2}-2 t t^{\prime} \cos \angle\left(V, V^{\prime}\right)
$$

and the inner product on $T_{Q} Y$ by

$$
<W, W^{\prime}>=t t^{\prime} \cos \angle\left(V, V^{\prime}\right) .
$$

We then have $|W|:=<W, W>^{1 / 2}=d_{T_{Q} Y}(0, W)$ where $0=S_{Q} Y \times\{0\}$ is the origin of the cone $T_{Q} Y$. The projection map $\pi: Y \rightarrow T_{Q} Y$ is defined by setting

$$
\pi(P)=([c], d(Q, P))
$$

where $c$ is the geodesic joining $Q$ to $P$ and $[c] \in S_{Q} Y$ is the equivalence class of $c$. The NPC condition guarantees that $\pi$ is a distance decreasing map. We refer to $[\mathrm{BH}]$ for more details.

\section{$2.3 \quad$ w-harmonic maps}

We first recall the Korevaar-Schoen energy for a map into a complete metric space (cf. $[\mathrm{EF}])$. Let $X$ be a 2-dimensional Riemannian complex and $d x$ the volume form defined by the Riemannian metric. Let $B_{\epsilon}(x)$ be the set of points in $X$ at a distance at most $\epsilon$ from $x$ and set $S_{\epsilon}(x)=$ $\partial B_{\epsilon}(x)$. Define $e_{\epsilon}: X \rightarrow \mathbf{R}$ by

$$
e_{\epsilon}(x)=\int_{y \in S_{\epsilon}(x)} \frac{d^{2}(f(x), f(y))}{\epsilon^{2}} \frac{d \sigma_{x, \epsilon}}{\epsilon}
$$

where $\sigma_{x, \epsilon}$ is the induced measure on $S_{\epsilon}(x)$. We define a family of functionals $E_{\epsilon}^{f}: C_{c}(X) \rightarrow \mathbf{R}$ by setting

$$
E_{\epsilon}^{f}(\varphi)=\int_{X} \varphi e_{\epsilon} d x
$$

We say $f$ has finite energy (or that $f \in W^{1,2}(X, Y)$ ) if

$$
E^{f}:=\sup _{\varphi \in C_{c}(\Omega), 0 \leq \varphi \leq 1} \limsup _{\epsilon \rightarrow 0} E_{\epsilon}^{f}(\varphi)<\infty .
$$


It can be shown that if $f$ has finite energy, the measures $e_{\epsilon}(x) d x$ converge weakly to a measure which is absolutely continuous with respect to the Lebesgue measure. Therefore, there exists a function $e(x)$, which we call the energy density, so that $e_{\epsilon}(x) d x \rightarrow e(x) d x$. In analogy to the case of smooth targets, we write $|\nabla f|^{2}(x)$ in place of $e(x)$. In particular, $E^{f}=\int_{X}|\nabla f|^{2} d x$.

Let $\Omega=F^{\text {int }}$ for some 2-simplex $F$ of $X$. For $V \in \Gamma \Omega$ where $\Gamma \Omega$ is the set of Lipschitz vector fields on $\Omega,\left|f_{*}(V)\right|^{2}$ is similarly defined. The real valued $L^{1}$ function $\left|f_{*}(V)\right|^{2}$ generalizes the norm squared on the directional derivative of $f$. The generalization of the pull-back metric is

$$
\pi_{f}(V, W)=\Gamma \Omega \times \Gamma \Omega \rightarrow L^{1}(\Omega, \mathbf{R})
$$

where

$$
\pi_{f}(V, W)=\frac{1}{2}\left|f_{*}(V+W)\right|^{2}-\frac{1}{2}\left|f_{*}(V-W)\right|^{2} .
$$

We refer to [KS1] for more details.

Let $X$ be a 2-dimensional admissible Euclidean complex and $Y$ an NPC space. An isometric action of a group $\Gamma=\pi_{1}(X)$ on $Y$ is a homomorphism $\rho: \Gamma \rightarrow \operatorname{Isom}(Y)$. A map $\tilde{\varphi}: \tilde{X} \rightarrow Y$ is said to be equivariant if

$$
\rho(\gamma) \tilde{\varphi}(p)=\tilde{\varphi}(\gamma p)
$$

for $\gamma \in \Gamma$ and $p \in X$. By identifying $X$ with a fundamental domain of $\tilde{X}$, we can think of $\tilde{\varphi}$ also being defined on $X$. We say $\tilde{\varphi}: \tilde{X} \rightarrow Y$ has finite energy if

$$
E^{\tilde{\varphi}}=\int_{X}|\nabla \tilde{\varphi}|^{2} d x<\infty .
$$

In order to include certain important examples appearing in $p$-adic geometry (e.g. $p$-adic buildings), we will assume that for each 2 -simplex $F$ in $X$, we have an associated weight $w$; more precisely $w$ assigns a positive number $w(F)$ to each 2-simplex $F$ of $X$. We define the $w$-measure $d \mu_{w}$ by setting

$$
d \mu_{w}=w(F) d x
$$

where $d x$ is the volume form on $F$ defined by metric $g_{F}$. We define the $w$-energy $E_{w}(\tilde{\varphi})$ of a finte energy map $\tilde{\varphi}: \tilde{X} \rightarrow Y$ as

$$
E_{w}(\tilde{\varphi})=\sum_{F} w(F) \int_{F}|\nabla \tilde{\varphi}|^{2} d x=\sum_{F} \int_{F}|\nabla \tilde{\varphi}|^{2} d \mu_{w}
$$

where $\sum_{F}$ indicates the sum over all $n$-dimensional simplices $F$ of $X$. For the sake of notational simplicity, we will fix weights $w(F)$ on $F$ and 
we will denote $d \mu=d \mu_{w}, E=E_{w}$, etc. A map $\tilde{f}: \tilde{X} \rightarrow Y$ is said to be $w$-harmonic if $E(\tilde{f}) \leq E(\tilde{\varphi})$ for all equivariant finite energy maps $\tilde{\varphi}: \tilde{X} \rightarrow Y$. If $w(F)=1$ for all $F$, then we recover the usual notion of harmonicity defined in [DM1].

\subsection{Blow up maps and tangent maps}

Let $X$ be a Euclidean admissible 2-complex $X, Y$ an NPC space and $f: X \rightarrow Y$ a $w$-harmonic map. Fix $p \in X$. We set $S t(p)=\operatorname{star}(p)$ if $p \in X^{(0)}, S t(p)=\operatorname{star}(E)$ if $p \in E-X^{(0)}$ and $S t(p)=F$ if $p \in F-X^{(1)}$. For any $\sigma>0$ sufficiently small so that $B_{\sigma}(p) \subset S t(p)$, let

$$
\begin{gathered}
E(\sigma)=\int_{B_{\sigma}(p)}|\nabla f|^{2} d \mu \\
I(\sigma)=\int_{\partial B_{\sigma}(p)} d^{2}(f, f(p)) d s
\end{gathered}
$$

and

$$
\mu(\sigma)=\sqrt{\frac{\sigma}{I(\sigma)}} .
$$

Let $B_{1}$ be a dilation of $B_{\sigma}(p)$ by factor of $\frac{1}{\sigma}$ and $d_{\sigma}: Y \times Y \rightarrow \mathbf{R}$ be the distance function on $Y$ defined by $d_{\sigma}(\cdot, \cdot)=\mu(\sigma) d(\cdot, \cdot)$. The $w$-measure $d \mu_{w}$ is inherited on $B_{1}$ from $B_{\sigma}(p)$ without any dilation. We define the $\sigma$-blow up map of $f$ at $p$ as the map

$$
f_{\sigma}: B_{1} \rightarrow\left(Y, d_{\sigma}\right)
$$

defined by

$$
f_{\sigma}(z)=f\left(\frac{z}{\sigma}\right)
$$

The following results for $w$-harmonic maps from a 2-complex into a non-positively metric space follow by minor modification of the arguments presented in [DM1]. (In [DM1], we only considered 2-simplices isometric to the standard equilateral triangle and a weight function $w$ so that $w(F)=1$ for all 2-simplices $F$ of $X$.)

Theorem 1 Let $f: X \rightarrow(Y, d)$ be a w-harmonic map from an Euclidean admissible 2-complex into an $N P C$ space $(Y, d)$. For each $p \in X$, the function.

$$
\sigma \mapsto \frac{\sigma E(\sigma)}{I(\sigma)}
$$


is monotone non-decreasing for $\sigma<\sigma_{0}$ where $\sigma_{0}$ is sufficiently small so that $B_{\sigma_{0}}(p) \subset S t(p)$. We call

$$
\operatorname{ord}(p)=\lim _{\sigma \rightarrow 0} \frac{\sigma E(\sigma)}{I(\sigma)}
$$

the order of $f$ at $p$. Let $\alpha=\operatorname{ord}(p)$. Then the functions

$$
\sigma \mapsto \frac{I(\sigma)}{\sigma^{1+2 \alpha}}
$$

and

$$
\sigma \mapsto \frac{E(\sigma)}{\sigma^{2 \alpha}}
$$

are monotone non-decreasing for $\sigma<\sigma_{0}$.

Theorem 2 Let $f: X \rightarrow(Y, d)$ be a w-harmonic map from an Euclidean admissible 2-complex into an NPC space $(Y, d)$. Fix $p \in X$ and let $\alpha=\operatorname{ord}(p)$. There exists a sequence $\sigma_{i} \rightarrow 0$ so that the $\sigma_{i}$-blow up maps $f_{\sigma_{i}}: B_{1} \rightarrow\left(Y, d_{\sigma_{i}}\right)$ of $f$ at $p$ converge in the sense of KorevaarSchoen (cf. [KS2]) to map $f_{*}: B_{1} \rightarrow\left(Y_{*}, d_{*}\right)$ into an NPC space. The map $f_{*}$ is Lipschitz continuous except possibly at the vertex and is homogeneous of order $\alpha$, i.e.

$$
d_{*}\left(f_{*}(z), f_{*}(0)\right)=|z|^{\alpha} d_{*}\left(f_{*}\left(\frac{z}{|z|}\right), f_{*}(0)\right)
$$

for every $z \in B_{1}$. We call $f_{*}$ a tangent map of $f$ at $p$.

We remark that one of the consequences of the convergence in the sense of Korevaar-Schoen is that the directional energy converges in $L^{1}$. In particular, if we consider $\partial B_{\lambda}$ as a graph, let $\tau$ be the arclength parameter on each edge, and consider $f_{\sigma_{i}}$ and $f_{*}$ as maps defined on $\partial B_{\lambda}$ by the restriction, then

$$
\int_{\partial B_{\lambda}}\left|\frac{\partial f_{\sigma_{i}}}{\partial \tau}\right|^{2} d s \rightarrow \int_{\partial B_{\lambda}}\left|\frac{\partial f_{*}}{\partial \tau}\right|^{2} d s \text { for a.e. } \lambda \in[0,1] .
$$

Here $d s$ is the measure induced on $\partial B_{\lambda}$ from the measure $d \mu_{w}$ on $B_{1}$.

\subsection{Existence and Regularity Results}

We also have the following existence and regularity of $w$-harmonic maps which again follow from a simple modifications of [DM1] 
Theorem 3 Let $X$ be an admissible Euclidean 2-complex with $\Gamma=$ $\pi_{1}(X), Y$ an NPC space and $\rho: \Gamma \rightarrow \operatorname{Isom}(Y)$ be an isometric action of $\Gamma$. Assume that $\rho$ does not fix an equivalent class of rays. If the curvature of $Y$ is strictly negative or $Y$ is locally compact, then there exists a $\rho$-equivariant w-harmonic map $\tilde{f}: \tilde{X} \rightarrow Y$.

Theorem 4 Let $X$ be an admissible Euclidean 2-complex, $Y$ an $N P C$ space and $f: X \rightarrow Y$ a w-harmonic map. Then $f$ is Lipschitz continuous away from the 0 -simplices of $X$ with the Lipschitz bound dependent only on the total $w$-energy of $f$ and the distance to the 0-simplices. Let $p$ be a 0 -simplex and $\alpha$ be the order of $f$ at $p$. Then there exists $\sigma>0$ so that

$$
|\nabla f|^{2}(q) \leq C r^{2 \alpha-2}
$$

for all $q \in B_{\sigma}(p)$ where $C$ depends on $E(f)$ and $r=d_{X}(p, q)$.

\section{The harmonicity of the energy density}

Let $X$ an admissible Euclidean 2-complex, $Y$ an NPC space and $f$ : $X \rightarrow Y$ be a $w$-harmonic map. In this section, we show that under the $\operatorname{assumption} \operatorname{ord}(p) \geq 1$ for every $p \in X^{(0)}$, we can prove that the energy density function is harmonic.

Fix a 1-simplex $E$ of $X$, let $p \in E$ and $\sigma_{0}>0$ sufficiently small so that $B_{\sigma_{0}}(p) \subset \operatorname{star}(E)$ if $p \in E-X^{(0)}$ or $B_{\sigma_{0}}(p) \subset \operatorname{star}(p)$ if $p$ is a 0 -simplex. Suppose $F$ is a 2 -simplex incident to $E$. Recall that there exists a simplical isometry $\phi_{F}:\left(F, g_{F}\right) \rightarrow T$ where $T \subset \mathbf{R}^{2}$ is an Euclidean triangle. Let $\psi$ be the linear isometry of $\mathbf{R}^{2}$ which takes $T=\phi_{F}(F)$ into $y \geq 0, \phi_{F}(E)$ into the line $y=0$ and $\phi_{F}(p)$ to the origin $(0,0)$. We refer to the coordinate $(x, y)$ of $F$ as the composition $\psi \circ \phi_{F}$. We will write $\left|\frac{\partial f_{F}}{\partial x}\right|^{2},\left|\frac{\partial f_{F}}{\partial y}\right|^{2}$ and $\frac{\partial f_{F}}{\partial x} \cdot \frac{\partial f_{F}}{\partial y}$ to denote $\left|f_{*}\left(\frac{\partial}{\partial x}\right)\right|^{2}$, $\left|f_{*}\left(\frac{\partial}{\partial y}\right)\right|^{2}$ and $\pi_{f}\left(\frac{\partial}{\partial x}, \frac{\partial}{\partial y}\right)$ respectively on $F$. These are $L^{1}$-functions on $F$.

Lemma 5 Let $p \in E-X^{(0)}, \sigma_{0}>0$ sufficiently small so that $B_{\sigma_{0}}(p) \subset$ $\operatorname{star}(E)$ and $D \subset \mathbf{R}^{2}$ be a disk of radius $\sigma_{0}$ centered at the origin. Define 
$\left|\partial_{x} f_{E}\right|^{2},\left|\partial_{y} f_{E}\right|^{2}: D \rightarrow \mathbf{R}$ by setting

$$
\begin{aligned}
\left|\partial_{x} f_{E}\right|^{2}(x, y) & =\left\{\begin{array}{cc}
\sum_{F \in \mathcal{F}(E)}\left|\frac{\partial f_{F}}{\partial x}\right|^{2}(x, y) & y \geq 0 \\
\sum_{F \in \mathcal{F}(E)}\left|\frac{\partial f_{F}}{\partial x}\right|^{2}(x,-y) & y<0
\end{array}\right. \\
\left|\partial_{y} f_{E}\right|^{2}(x, y) & =\left\{\begin{array}{cc}
\sum_{F \in \mathcal{F}(E)}\left|\frac{\partial f_{F}}{\partial y}\right|^{2}(x, y) & y \geq 0 \\
\sum_{F \in \mathcal{F}(E)}\left|\frac{\partial f_{F}}{\partial y}\right|^{2}(x,-y) & y<0
\end{array}\right.
\end{aligned}
$$

respectively, where $\mathcal{F}(E)$ is the set of 2-simplices incident to $E$. The functions $\left|\partial_{x} f_{E}\right|^{2}$ and $\left|\partial_{y} f_{E}\right|^{2}$ are weakly subharmonic in $D$, i.e.

$$
\int_{D} \triangle \eta\left|\partial_{x} F_{E}\right|^{2} \geq 0 \text { and } \int_{D} \triangle \eta\left|\partial_{y} F_{E}\right|^{2} \geq 0
$$

for every $\eta \in C_{c}^{\infty}(D)$.

Proof. Fix $\epsilon>0$ and let $f_{0}(x, y)=f(x, y)$ and $f_{1}(x, y)=f(x+\epsilon, y)$ on each face $F \in \mathcal{F}(E)$. For non-negative smooth function $\eta$ with compact support in $D$, let $f_{\eta}=(1-\eta) f_{0}+\eta f_{1}$. We can follow the proof of Lemma 2.4.2 and Remark 2.4.3 of [KS1] (also see [DM1], Proposition 3.8) to see that

$$
\int_{D} \triangle \eta d^{2}(f(x, y), f(x+\epsilon, y)) \geq 0 .
$$

Divide by $\epsilon^{2}$ and use the fact that $f$ is Lipschitz as well as the Dominated Convergence Theorem as we let $\epsilon \rightarrow 0$ to see that $\left|\partial_{x} f_{E}\right|^{2}$ is weakly subharmonic.

The function

$$
\phi_{F}=\left|\frac{\partial f_{F}}{\partial x}\right|^{2}-\left|\frac{\partial f_{F}}{\partial y}\right|^{2}-2 i \frac{\partial f_{F}}{\partial x} \cdot \frac{\partial f_{F}}{\partial x}
$$

is holomorphic in $F$ and $\operatorname{Im} \sum_{F \in \mathcal{F}(E)} \phi_{F}(x, 0)=0$ ([DM1], Theorem 3.9). Therefore, the function $\phi_{E}: D \rightarrow \mathbf{C}$ defined by

$$
\phi_{E}(x, y)=\left\{\begin{array}{cc}
\sum_{F \in \mathcal{F}(E)} \phi_{F}(x, y) & y \geq 0 \\
\sum_{F \in \mathcal{F}(E)} \overline{\phi_{F}}(x,-y) & y<0
\end{array}\right.
$$


is holomorphic by the reflection principle. This implies that $\left|\partial_{x} f_{E}\right|^{2}-$ $\left|\partial_{y} f_{E}\right|^{2}$ is harmonic. Since $\left|\partial_{x} f_{E}\right|^{2}$ is weakly subharmonic, $\left|\partial_{y} f_{E}\right|^{2}$ is also weakly subharmonic. Q.E.D.

Lemma 6 Let $p \in E-X^{(0)}$ and $D$ as before. The function $e_{E}: D \rightarrow \mathbf{R}$ defined by setting

$$
e_{E}(x, y)=\left\{\begin{array}{cc}
\sum_{F \in \mathcal{F}(E)}\left|\nabla f_{F}\right|^{2}(x, y) & y \geq 0 \\
\sum_{F \in \mathcal{F}(E)}\left|\nabla f_{F}\right|^{2}(x,-y) & y<0
\end{array}\right.
$$

is weakly subharmonic

Proof. Since $e_{E}=\left|\partial_{x} f_{E}\right|^{2}+\left|\partial_{y} f_{E}\right|^{2}$, the assertion follows from Lemma 5. Q.E.D.

Lemma 7 Let $p \in E$. For any $F \in \mathcal{F}(E)$, let $(r, \theta)$ be polar coordinates for $F$ centered at $p \in E$ so that $E-\{p\}$ is given by the line $\theta=0$ and the line $\theta=\pi$. Let $r_{1}, r_{2}, \theta_{1}, \theta_{2}$ be so that $R_{F}=\left\{(r, \theta): 0<r_{1} \leq r \leq\right.$ $\left.r_{2}, \theta_{1} \leq \theta \leq \theta_{2}\right\} \subset E \cup(F-\partial F)$ for all $F \in \mathcal{F}(E)$. Then

$$
(r, \theta) \mapsto \sum_{F \in \mathcal{F}(E)}\left|\nabla f_{F}\right|^{2}(r, \theta)
$$

for $0<r_{1} \leq r \leq r_{2}$ and $\theta_{1} \leq \theta \leq \theta_{2}$ is a $W^{1,2}$ function.

Proof. If $R_{F}$ is contained in $F-\partial F$, then the assertion is obvious since the weak subharmonicity and boundedness of $\left|\nabla f_{F}\right|^{2}$ implies $\left|\nabla f_{F}\right|^{2}$ is $W_{l o c}^{1,2}$. If $p \in E-X^{(0)}$, then this is also obvious by Lemma 6 . Now we assume $p$ is a 0 -simplex. We need to consider the case when $\theta_{1}=0$. Let $r_{0} \in\left(r_{1}, r_{2}\right)$ and $p^{\prime} \in E$ be so that $d\left(p, p^{\prime}\right)=r_{0}$. Let $D$ be defined as before with $p$ replaced by $p^{\prime}$. Then the subharmonicity of $e_{E}$ in Lemma 6 implies

$$
(r, \theta) \mapsto \sum_{F \in \mathcal{F}(E)}\left|\nabla f_{F}\right|^{2}(r, \theta)
$$

is $W_{l o c}^{1,2}$ in $D \cap\left\{(r, \theta): 0<r_{1} \leq r \leq r_{2}, 0 \leq \theta \leq \theta_{2}\right\}$. Thus, assertion follows immediately. Q.E.D. 
Corollary 8 For $p \in X^{(1)}$, let $\sigma_{0}>0$ be so that $B_{\sigma_{0}}(p) \subset \operatorname{star}(E)$ if $p \in X^{(1)}-X^{(0)}$ and $B_{\sigma_{0}}(p) \subset \operatorname{star}(p)$ if $p$ is a 0 -simplex. Then

$$
r \mapsto \int_{\partial B_{r}(p)}|\nabla f|^{2} d s
$$

for $0<r<\sigma_{0}$ is a $W_{\text {loc }}^{1,2}$ function.

Proof. Let $(r, \theta)$ be the polar coordinates in Lemma 7. If $p \in$ $X^{(1)}-X^{(0)}$ then the conclusion follows immediately from Lemma 7 and the fact that

$$
\int_{\partial B_{r}(p)}\left|\nabla f_{F}\right|^{2} d s=\sum_{F \in \mathcal{F}(E)} \int_{0}^{\pi}\left|\nabla f_{F}\right|^{2}(r, \theta) r d \theta .
$$

If $p$ is a 0 -simplex, then for every 1 -simplex $E$ with $p \in E$, there exists $\theta_{2}>0$ sufficiently small so that

$$
(t, \theta) \mapsto \sum_{F \in \mathcal{F}(E)}\left|\nabla f_{F}\right|^{2}(t, \theta)
$$

is $W_{l o c}^{1,2}$ for $0<t<\sigma_{0}$ and $0 \leq \theta \leq \theta_{2}$ by Lemma 7 . For any $r \in\left(0, \sigma_{0}\right)$, let $q \in E$ so that $d(p, q)=r$. There exists $\epsilon>0$ small so that

$$
B_{\epsilon}(q) \subset \cup\left\{(t, \theta) \in F: t \in(r-\epsilon, r+\epsilon), \theta \in\left(0, \theta_{2}\right)\right\} .
$$

Thus,

$$
t \mapsto \int_{\partial B_{t}(p) \cap B_{\epsilon}(q)}|\nabla f|^{2} d s=\sum_{F \in \mathcal{F}(E)} \int_{0}^{\theta_{2}(t)}\left|\nabla f_{F}\right|^{2}(t, \theta) t d \theta
$$

is $W^{1,2}$ where $\theta_{2}(t)=\sup \left\{\theta:(t, \theta) \in B_{\epsilon}(q)\right\}$. This together with the fact that $\left|\nabla f_{F}\right|^{2}$ is $W^{1,2}$ in the interior of a 2 -simplex $F$ implies the assertion. Q.E.D.

Corollary 8 implies that

$$
r \mapsto \int_{\partial B_{r}(p)}|\nabla f|^{2} d s
$$

is absolutely continuous and hence differentiable a.e. for $\sigma \in\left[0, \sigma_{0}\right]$. 
Lemma 9 Let $f: X \rightarrow Y$ be a harmonic map, $p \in X^{(0)}$ and $\operatorname{ord}(p) \geq 1$. For every $\sigma_{0}$ so that $B_{\sigma_{0}}(p) \subset \operatorname{star}(p)$, there exists a set $A \subset\left[0, \sigma_{0}\right]$ of positive measure so that

$$
\frac{d}{d \sigma}\left(\frac{1}{\sigma} \int_{\partial B_{\sigma}(p)}|\nabla f|^{2} d s\right) \geq 0 \text { for } \sigma \in A .
$$

Proof. Let

$$
G(\sigma)=\frac{1}{\sigma} \int_{\partial B_{\sigma}(p)}|\nabla f|^{2} d s
$$

and suppose there exists $\sigma_{0}>0$ so that $G^{\prime}(\sigma)<0$ for almost all $0<\sigma<$ $\sigma_{0}$. Then $G(\sigma)$ is non-increasing for $0<\sigma<\sigma_{0}$. Let $c_{1}=\lim _{\sigma \rightarrow 0} G(\sigma)$. Now let

$$
F(\sigma)=\frac{2}{\sigma^{2}} \int_{B_{\sigma}(p)}|\nabla f|^{2} d \mu
$$

The assumption that $\operatorname{ord}(p) \geq 1$ and Theorem 1 implies that $F(\sigma)$ is non-decreasing for all $0<\sigma<\sigma_{0}$. Let $c_{2}=\lim _{\sigma \rightarrow 0} F(\sigma)$. We claim $G(\sigma) \geq F(\sigma)$. Indeed,

$$
\begin{aligned}
0 & \leq\left(\frac{2}{\sigma^{2}} \int_{B_{\sigma}(p)}|\nabla f|^{2} d \mu\right)^{\prime} \\
& =\frac{2}{\sigma^{2}} \int_{\partial B_{\sigma}(p)}|\nabla f|^{2} d \mu-\frac{4}{\sigma^{3}} \int_{B_{\sigma}(p)}|\nabla f|^{2} d \mu \\
& =\frac{2}{\sigma}(G(\sigma)-F(\sigma)) .
\end{aligned}
$$

Since $G(\sigma)$ is non-increasing, $F(\sigma)$ is non-decreasing and $G(\sigma) \geq F(\sigma)$, we conclude $c_{1}>c_{2}$ and there exists $\sigma_{1}$ so that $G(\sigma)>\frac{c_{1}+c_{2}}{2}>F(\sigma)$ for $0<\sigma \leq \sigma_{1}$. Therefore,

$$
\begin{aligned}
& \frac{c_{1}+c_{2}}{2} \frac{\sigma_{1}^{2}}{2} \\
& >\int_{B_{\sigma_{1}}(p)}|\nabla f|^{2} d \mu \\
& =\int_{0}^{\sigma_{1}}\left(\int_{\partial B_{\sigma}(p)}|\nabla f|^{2} d s\right) d \sigma \\
& >\int_{0}^{\sigma_{1}} \frac{c_{1}+c_{2}}{2} \sigma d \sigma=\frac{c_{1}+c_{2}}{2} \frac{\sigma_{1}^{2}}{2},
\end{aligned}
$$

a contradiction. Q.E.D. 
Lemma 10 Let $f: X \rightarrow Y$ be a harmonic map, $p \in X^{(0)}$ and $\operatorname{ord}(p)>$ 1. Either $f$ is constant on star $(p)$ or there exists a set $A^{\prime} \subset\left[0, \sigma_{0}\right]$ of positive measure so that

$$
\frac{d}{d \sigma}\left(\frac{1}{\sigma} \int_{\partial B_{\sigma}(p)}|\nabla f|^{2} d s\right) d s>0 \text { for } \sigma \in A^{\prime},
$$

where $\sigma_{0}$ is sufficiently small so that $B_{\sigma_{0}}(p) \subset \operatorname{star}(p)$.

Proof. If $\alpha:=\operatorname{ord}(p)>1$, then Theorem 1 implies that $\lim _{\sigma \rightarrow 0} F(\sigma)=$ 0 . Thus, for any $\epsilon>0$, there exists $\sigma_{1}>0$ so that $F\left(\sigma_{1}\right)<\epsilon$. Thus, there exists $\sigma_{2} \in\left(\sigma_{1} / 2, \sigma_{1}\right)$ so that

$$
\begin{aligned}
\frac{1}{\sigma_{2}} \int_{\partial B_{\sigma_{2}}(p)}|\nabla f|^{2} d s & \leq \frac{2}{\sigma_{1}} \int_{\partial B_{\sigma_{2}}(p)}|\nabla f|^{2} d s \\
& \leq\left(\frac{2}{\sigma_{1}}\right)^{2} \int_{\sigma_{1} / 2}^{\sigma_{1}}\left(\int_{\partial B_{\sigma}(p)}|\nabla f|^{2} d s\right) d \sigma \\
& <2 \epsilon,
\end{aligned}
$$

which implies $\lim _{\sigma \rightarrow 0} G(\sigma)=0$. Thus, unless $G(\sigma)$ is identically equal to $0, G^{\prime}(\sigma)>0$ for $\sigma \in A^{\prime}$ where $A^{\prime} \subset(0, \sigma)$ is of positive measure. But if $G(\sigma)$ is identically zero then $F(\sigma)$ is identically equal to 0 which implies $|\nabla f|^{2}(p)=0 p \in B_{\sigma_{0}}(p)$ and hence $f$ is constant in $B_{\sigma_{0}}(p)$. By following the proof of [GS] Proposition 3.4, it is not hard to show $f$ is constant in $\operatorname{star}(p)$. Q.E.D.

Lemma 11 Let $p \in E-X^{(0)}$. For $\sigma_{0}>0$ so that $B_{\sigma_{0}}(p) \subset \operatorname{star}(E)$,

$$
\frac{d}{d \sigma}\left(\frac{1}{\sigma} \int_{\partial B_{\sigma}(p)}|\nabla f|^{2} d s\right) \geq 0 \text { for } 0<\sigma<\sigma_{0} .
$$

ProOF. For $0<\sigma<\sigma_{0}$,

$$
\int_{\partial B_{\sigma}(p)}|\nabla f|^{2} d s=\int_{\partial D_{\sigma}(x, 0)} e_{E} d s
$$

where $(x, 0)$ is the coordinates for $p$ and $D_{\sigma}(x, 0)$ is a disk of radius $\sigma$ centered at $(x, 0)$. The conclusion follows immediately from the subharmonicity of $e_{E}$. Q.E.D. 
Theorem 12 If $f: X \rightarrow Y$ is a w-harmonic map so that $\operatorname{ord}(p) \geq 1$ for all $p \in X^{(0)}$, then $|\nabla f|^{2}$ is a harmonic function on each 2-simplex $F$ of $X$. If $\operatorname{ord}(p)>1$ for all $p \in X^{(0)}$, then $f$ is a constant map.

Proof. Let $p \in X^{(0)}$. Since $\partial X=\emptyset$, there exists $\sigma_{0}>0$ so that $B_{\sigma_{0}}(p) \subset \operatorname{star}(p)$. By Lemma 9 , we can choose $\delta_{k}^{p} \rightarrow 0$ so that

$$
\left.\frac{d}{d \sigma}\left(\frac{1}{\sigma} \int_{\partial B_{\sigma}(p)}|\nabla f|^{2} d s\right)\right|_{\sigma=\delta_{k}^{p}} \geq 0 .
$$

Fix $k$ and let $\epsilon>0$. Choose points $\left\{p_{1}, \ldots, p_{n}\right\} \subset X^{(1)}-\cup_{p \in \mathcal{V}(X)} B_{\delta_{k}^{p}}(p)$ and positive real numbers $\left\{r_{1}, \ldots, r_{n}\right\}$ so that $(1) r_{i}<\epsilon,(2) B_{r_{i}}\left(p_{i}\right) \cap$ $B_{r_{j}}\left(p_{j}\right)=\emptyset$ for $i, j=1, \ldots, n$ and $i \neq j,(3) B_{\delta_{k}^{p}}(p) \cap B_{r_{i}}\left(p_{i}\right)=\emptyset$ for all $p \in X^{(0)}$ and $i=1, \ldots, n$ and (4) $X^{(1)}$ is covered by

$$
\left(\bigcup_{p \in X^{(0)}} \overline{B_{\delta_{k}^{p}}(p)}\right) \cup\left(\bigcup_{i=1}^{n} \overline{B_{r_{i}}\left(p_{i}\right)}\right) .
$$

Let $X^{\prime}=X-\left(\bigcup_{p \in X^{(0)}} \overline{B_{\delta_{k}^{p}}(p)}\right) \cup\left(\bigcup_{i=1}^{n} \overline{B_{r_{i}}\left(p_{i}\right)}\right)$. For each $F \in \mathcal{F}(X)$, we have

$$
-\int_{F} \nabla|\nabla f|^{2} \cdot \nabla \zeta \geq 0
$$

for any $\zeta \in C_{c}^{\infty}(F)$. Let $\zeta$ approximate the characteristic function of $F^{\prime}=F \cap X^{\prime}$. Then

$$
\begin{aligned}
0 & \geq-\int_{\partial F^{\prime}} \frac{\partial}{\partial \eta}|\nabla f|^{2} d s \\
= & \sum_{p \in X^{(0)} \cap F} \int_{\partial B_{\delta_{k}^{p}}(p) \cap F} \frac{\partial}{\partial r}|\nabla f|^{2} d s+\sum_{p_{i} \in F} \int_{\partial B_{r_{i}}\left(p_{i}\right) \cap F} \frac{\partial}{\partial r}|\nabla f|^{2} d s \\
= & \left.\sum_{p \in X^{(0)} \cap F} \delta_{k}^{p} \frac{d}{d \sigma}\left(\frac{1}{\sigma} \int_{\partial B_{\delta_{k}^{p}}(p)}|\nabla f|^{2} d s\right)\right|_{\sigma=\delta_{k}^{p}} \\
& \quad+\left.\sum_{p_{i} \in F} r_{i} \frac{d}{d \sigma}\left(\frac{1}{\sigma} \int_{\partial B_{\sigma}\left(p_{i}\right)}|\nabla f|^{2} d s\right)\right|_{\sigma=r_{i}}
\end{aligned}
$$

where $\eta$ is the outward pointing normal to $\partial F^{\prime}$ defined everywhere except at finite number of points. On the other hand,

$$
-\sum_{F \in \mathcal{F}(X)} \int_{\partial F^{\prime}} \frac{\partial}{\partial \eta}|\nabla f|^{2} d s
$$




$$
\begin{aligned}
= & \left.\sum_{p \in X^{(0)}} \delta_{k}^{p} \frac{d}{d \sigma}\left(\frac{1}{\sigma} \int_{\partial B_{\delta_{k}^{p}}(p)}|\nabla f|^{2} d s\right)\right|_{\sigma=\delta_{k}^{p}} \\
& +\left.\sum_{i=1}^{n} r_{i} \frac{d}{d \sigma}\left(\frac{1}{\sigma} \int_{\partial B_{\sigma}\left(p_{i}\right)}|\nabla f|^{2} d s\right)\right|_{\sigma=r_{i}} \\
\geq 0, &
\end{aligned}
$$

where the last inequality is implied by Lemma 11 and (3). Thus,

$$
\int_{\partial F^{\prime}} \frac{\partial}{\partial \eta}|\nabla f|^{2} d s=0
$$

The arbitrariness of $\epsilon$ along with the fact that $\delta_{i}^{p} \rightarrow 0$ proves the harmonicity of $|\nabla f|^{2}$ on each $F$.

If $\operatorname{ord}(p)>1$ for all $p \in X^{(0)}$, then either $f$ is a constant map or $\delta_{k}^{p}$ can be chosen so that (3) is given with a strict inequality for some $p \in X^{(0)}$ by Lemma 10 . The strict inequality in (3) leads to a strict inequality in (5) which contradicts (4). Thus, $f$ is a constant map. Q.E.D.

Theorem 13 If $f: X \rightarrow Y$ is a harmonic map with ord $(p) \geq 1$ for all $p \in X^{(0)}$, then $f$ is totally geodesic on each 2-simplex $F$ of $X$, i.e. $f$ maps every Euclidean line in $F$ to a geodesic in $Y$. If the curvature of $Y$ is strictly negative, then $f$ maps each 2-simplex $F$ into a geodesic. If $\operatorname{ord}(p)>1$ for all $p \in X^{(0)}$, then $f$ is constant.

Proof. By Theorem 12, $|\nabla f|^{2}=\left|\frac{\partial f}{\partial x}\right|^{2}+\left|\frac{\partial f}{\partial y}\right|^{2}$ is a harmonic function on each 2-simplex $F$ of $X$. Since $\phi_{F}$ defined by (2) is holomorphic, $\operatorname{Re} \phi_{F}=\left|\frac{\partial f}{\partial x}\right|^{2}-\left|\frac{\partial f}{\partial y}\right|^{2}$ is also a harmonic function. Thus, $\left|\frac{\partial f}{\partial x}\right|^{2}$ and $\left|\frac{\partial f}{\partial y}\right|^{2}$ are harmonic functions and hence smooth. Additionally, $\frac{1}{2} \operatorname{Im} \phi_{F}=$ $\frac{\partial f}{\partial x} \cdot \frac{\partial f}{\partial y}$ is harmonic and smooth. Let

$$
\left(\pi_{i j}\right)=\left(\begin{array}{cc}
\left|\frac{\partial f}{\partial x}\right|^{2} & \frac{\partial f}{\partial x} \cdot \frac{\partial f}{\partial y} \\
\frac{\partial f}{\partial x} \cdot \frac{\partial f}{\partial y} & \left|\frac{\partial f}{\partial y}\right|^{2}
\end{array}\right)
$$

and $\Omega$ be any open subset of $F$ where $\pi$ is non-degenerate. We will show that $\left.f\right|_{\Omega}$ is a totally geodesic map. First, we note that $(\Omega, \pi)$ is a smooth Riemannian manifold of non-positive curvature (see appendix). Let $d_{\pi}$ be the distance function induced by the metric $\pi$. The identity map $f_{0}: \Omega \rightarrow(\Omega, \pi)$ is a (smooth) harmonic map. Indeed, if $h_{0}: \Omega \rightarrow(F, \pi)$ is so that $E\left(h_{0}\right)<E\left(f_{0}\right)$ and $\left.h_{0}\right|_{\partial \Omega}=\left.f_{0}\right|_{\partial \Omega}$, 
then $E\left(f \circ h_{0}\right)<E\left(f \circ f_{0}\right)=E(f)$, a contradiction. Furthermore, $\triangle\left|\nabla f_{0}\right|^{2}=\triangle|\nabla f|^{2}=0$, so the Eells-Sampson Bochner's formula implies that $\left|\nabla d f_{0}\right|^{2}=0$ and $f_{0}$ is a totally geodesic map. Thus, if $t \mapsto \gamma(t)$ is a unit speed parameterization of a Euclidean line in $\Omega$, then $\gamma(t)$ is a geodesic with respect to the metric $\pi$. Hence, the Christoffel symbols of $\pi$ are identically constant which implies that $\pi$ is constant. In particular, this means that $\pi$ is flat, $t \mapsto \pi\left(\gamma^{\prime}(t), \gamma^{\prime}(t)\right)$ is constant, and given two constant speed parameterization $\gamma_{1}(t)$ and $\gamma_{2}(t)$ of a Euclidean line emanating from the same point, $t \mapsto d_{\pi}\left(\gamma_{1}(t), \gamma_{2}(t)\right)$ is a linear function. For any $z_{0} \in \Omega$, let $(r, \theta)$ be the polar coordinates centered at $z_{0}$. For any $0<r<R$ with $R$ sufficiently small, we therefore have

$$
\left|\frac{\partial f}{\partial r}\right|^{2}(r, \theta)=\frac{1}{R^{2}}\left(d_{\pi}\left((R, \theta), z_{0}\right)\right)^{2}
$$

and

$$
\left|\frac{\partial f}{\partial \theta}\right|^{2}(r, \theta)=\frac{r^{2}}{R^{2}}\left|\frac{\partial f}{\partial \theta}\right|^{2}(R, \theta) .
$$

Define $h: D_{R}\left(z_{0}\right) \rightarrow Y$ by setting

$$
h(r, \theta)=\left(1-\frac{r}{R}\right) f\left(z_{0}\right)+\frac{r}{R} f(R, \theta) .
$$

Note that $h$ maps radial lines to geodesic lines. The NPC condition implies that

$$
d\left(h\left(r, \theta_{1}\right), h\left(r, \theta_{2}\right)\right) \leq \frac{r}{R} d\left(f\left(R, \theta_{1}\right), f\left(R, \theta_{2}\right)\right) .
$$

Thus,

$$
\left|\frac{\partial h}{\partial \theta}\right|^{2}(r, \theta) \leq \frac{r^{2}}{R^{2}}\left|\frac{\partial f}{\partial \theta}\right|^{2}(R, \theta)=\left|\frac{\partial f}{\partial \theta}\right|^{2}(r, \theta) .
$$

Furthermore, the fact that the distance function induced by the pull back metric always bounds the pull back of the distance function implies

$$
\left|\frac{\partial h}{\partial r}\right|^{2}(r, \theta)=\frac{1}{R^{2}} d^{2}(f(R, \theta), f(0)) \leq \frac{1}{R^{2}}\left(d^{\pi}\left((R, \theta), z_{0}\right)\right)^{2}=\left|\frac{\partial f}{\partial r}\right|^{2}(r, \theta)
$$

This implies $E^{h} \leq E^{f}$, but since $f$ is energy minimizing $h=f$. Therefore, $f$ maps radial lines emanating from $z_{0}$ to geodesics. Since $z_{0}$ is an arbitrary point in $\Omega$, this proves $\left.f\right|_{\Omega}$ is totally geodesic in $\Omega$.

Since $\pi_{i j}$ is smooth, the set of points in $F$ where $\pi$ is non-degenerate is an open set. On the other hand, the above argument shows that $\pi$ 
is constant on this set, so this set is also closed. Thus, either $\pi$ is nondegenerate on all of $F$ or degenerate on all of $F$. In the former case, we are done by the argument in the previous paragraph. If $Y$ has strictly negative curvature, this case is impossible. In the latter case, we choose local coordinates so that the Hopf differential is equal to $d z^{2}$, i.e.

$$
\left|\frac{\partial f_{F}}{\partial x}\right|^{2}-\left|\frac{\partial f_{F}}{\partial y}\right|^{2}-2 i \frac{\partial f_{F}}{\partial x} \cdot \frac{\partial f_{F}}{\partial y}=1 .
$$

If $\pi$ not equal to the zero matrix in a neighborhood, then it follows that

$$
\left|\frac{\partial f_{F}}{\partial x}\right|^{2}=1 \text { and }\left|\frac{\partial f_{F}}{\partial y}\right|^{2}=0 .
$$

This immediately implies that $f$ maps this neighborhood to a Lipschitz curve. By the same argument as in the non-degenerate case, this line must be a geodesic. The last statement of the theorem follows from Theorem 12. Q.E.D.

\section{The first eigenvalue and order}

We now wish to establish assumptions on $X$ for which the order of the $w$-harmonic map at a 0 -simplex in $X$ is $\geq 1$. In this section, we define the first eigenvalue of a link of a 0 -simplex of $X$ with values in an NPC space and give a lower bound of the order in terms of the lower bound on the first eigenvalues.

Let $G$ be a metric graph. We denote the edges of $G$ by $e_{1}, \ldots, e_{L}$ and assume that each edge $e_{l}$ has an associated weight $\hat{w}_{l}=\hat{w}\left(e_{l}\right)$. In the case $G=L k(p)$ where $p \in X^{(0)}, X$ is an Euclidean admissible 2-complex with weight $w$, there is a one-to-one correspondence between the edges and the 2-simplices $F_{1}, . ., F_{L}$ incident to $p$; namely, $F_{l}$ is the join (i.e. convex hull) of $v$ and $e_{l}$. Moreover, the length of the edge $e_{l}$ is equal to the angle at vertex $p$ in $F_{l}$. We define

$$
\hat{w}\left(e_{l}\right)=w\left(F_{l}\right) .
$$

Returning to the case of a general metric graph $G$ with weights $\hat{w}_{l}$, $l=1, \ldots, L$, we define a measure $\hat{w}_{l} d \tau$ on each edge $e_{l}$ where $\tau$ is the arclength parameter of the edge. Let $d s$ be the measure on $G$ so that $\left.d s\right|_{e_{l}}=\hat{w}_{l} d \tau$. 
Let $T$ be an NPC space. A center of mass of a map $\varphi \in L^{2}(G, T)$ is a point $\bar{\varphi} \in T$ so that

$$
\int_{G} d_{T}^{2}(\varphi, \bar{\varphi}) d s=\inf _{P \in T} \int_{G} d_{T}^{2}(\varphi, P) d s .
$$

The unique existence of such a point is guaranteed by the NPC condition (cf. [KS1] Proposition 2.5.4). Now let $\mathcal{G}(T)$ be the set of Lipschitz functions $\varphi: G \rightarrow T$ into an NPC space $T$ and define the first eigenvalue of $G$ with values in $T$ as

$$
\lambda_{1}(G, T)=\inf _{\mathcal{G}(T)} \frac{\int_{G}\left|\frac{d \varphi}{d \tau}\right|^{2} d s}{\int_{G} d^{2}(\varphi, \bar{\varphi}) d s} .
$$

In the application, the NPC space $T$ will be a tangent cone of an NPC space $Y$. We will need the following lemma.

Lemma 14 Suppose $f: X \rightarrow Y$ is a continuous map and $Q \in Y$ so that

$$
\int_{\partial B_{\sigma}(p)} d^{2}(f, Q) d s=\inf _{P \in Y} \int_{\partial B_{\sigma}(p)} d^{2}(f, P) d s .
$$

If $\pi: Y \rightarrow T_{Q} Y$ is the projection map into the tangent cone of $Y$ at $Q$, then

$$
\int_{\partial B_{\sigma}(p)} d_{T_{Q} Y}^{2}(\pi \circ f, 0) d s=\inf _{V \in T_{Q} Y} \int_{\partial B_{\sigma}(p)} d_{T_{Q} Y}^{2}(\pi \circ f, V) d s
$$

where 0 is the origin of $T_{Q} Y$.

Proof. Let $t \mapsto c(t)$ be a geodesic so that $c(0)=Q$. By the minimizing property of $c(0)=Q$, we have

$$
0 \leq \int_{\partial B_{\sigma}(p)} d^{2}(f, c(t)) d s-\int_{\partial B_{\sigma}(p)} d^{2}(f, c(0)) d s .
$$

Furthermore, by Bridson-Haeflinger, Corollary II 3.6, we have

$$
\lim _{t \rightarrow 0} \frac{d(f, c(t))-d(f, c(0))}{t}=-\cos \angle\left(c, \gamma_{y}\right)
$$

where $\gamma_{y}$ is the geodesic from $c(0)$ to $f(y)$ and $\angle\left(\gamma_{y}, c\right)$ is the angle between $\gamma_{y}$ and $c$ at $c(0)=Q$. Therefore,

$$
\begin{aligned}
0 & \leq \lim _{t \rightarrow 0} \int_{\partial B_{\sigma}(p)} \frac{d^{2}(f, c(t))-d^{2}(f, c(0))}{t} d t \\
& =\lim _{t \rightarrow 0} \int_{\partial B_{\sigma}(p)} \frac{d(f, c(t))-d(f, c(0))}{t}(d(f, c(t))+d(f, c(0))) d s \\
& =-2 \int_{y \in \partial B_{\sigma}(p)} \cos \angle\left(\gamma_{y}, c\right) d(f(y), c(0)) d s .
\end{aligned}
$$


Let $[c]$ be the equivalence class of $c$ and $V=([c], 1) \in T_{Q} Y$. Since $\pi \circ \gamma_{y}$ is the (radial) geodesic from the origin 0 and $\pi \circ f(y)$ in $T_{Q} Y$,

$$
\cos \angle\left(\gamma_{y}, c\right) d(f(y), f(0))=<\pi \circ f(y), V>,
$$

and thus

$$
0 \leq-\int_{y \in \partial B_{\sigma}(p)}<\pi \circ f(y), V>d s .
$$

By the continuity of the inner product, (7) holds for all $V=\left(V_{0}, t\right) \in$ $T_{Q} Y$ where $V_{0}=V /|V|$. Therefore, for $t \geq 0$,

$$
\begin{aligned}
& \int_{\partial B_{\sigma}(p)} d_{T_{Q} Y}^{2}\left(\pi \circ f(y),\left(V_{0}, t\right)\right) d s \\
& \quad=\int_{\partial B_{\sigma}(p)} t^{2}+|\pi \circ f(y)|^{2}-2 t<\pi \circ f(y), V_{0}>d s \\
& \quad \geq \int_{\partial B_{\sigma}(p)}|\pi \circ f(y)|^{2} d s \\
& \quad=\int_{\partial B_{\sigma}(p)} d_{T_{Q} Y}^{2}(\pi \circ f(y), 0) d s .
\end{aligned}
$$

Q.E.D.

For $p \in X^{(0)}$ and $\sigma>0$ sufficiently small so that $B_{\sigma}(p) \subset \operatorname{star}(p)$, define

$$
\sigma: B_{1}(p) \rightarrow B_{\sigma}(p), \sigma(x)=\sigma x
$$

to be the dilation by $\sigma$ as was done in defining the domain of the blow up maps $f_{\sigma}$ (cf section 2.4). Since the edge $e_{l}$ of $L k(p)$ are isometrically identified with the interval $[0, \theta]$ where $\theta$ is the angle of $p$ in $F_{l}, \partial B_{1}$ is isometrically identified with $L k(p)$. Thus, Lemma 14 immediately implies

Corollary 15 Suppose $f: X \rightarrow Y$ is a Lipschitz map and $Q \in Y$ so that

$$
\int_{\partial B_{\sigma}(p)} d^{2}(f, Q) d s=\inf _{P \in Y} \int_{\partial B_{\sigma}(p)} d^{2}(f, P) d s .
$$

If $\pi: Y \rightarrow T_{Q} Y$ is the projection map into the tangent cone of $Y$ at $Q$, then

$$
\frac{\int_{L k(v)}\left|\frac{\partial(\pi \circ f \circ \sigma)}{\partial \tau}(x)\right|^{2} d s}{\int_{L k(v)}|\pi \circ f \circ \sigma(x)|^{2} d s} \geq \lambda_{1}\left(L k(v), T_{Q} Y\right) .
$$


Proof. By Lemma 14, the center of mass of the map $\pi \circ f \circ \sigma$ is 0 . Thus, the assertion follows immediately from the definition of $\lambda_{1}\left(L k(v), T_{Q} Y\right)$. Q.E.D.

A consequence of Corollary 15 is the following theorem which associates the first eigenvalue with the order of a $w$-harmonic map.

Theorem 16 Let $f: X \rightarrow Y$ be a w-harmonic map. If $\lambda_{1}\left(L k(p), T_{Q} Y\right) \geq$ $\beta$ (> $\beta$ ) for $p \in X^{(0)}$ and all $Q \in Y$, then $\alpha:=\operatorname{ord}(p) \geq \sqrt{\beta}(>\sqrt{\beta})$.

Proof. Let $\sigma_{i} \rightarrow 0$ so that $f_{\sigma_{i}} \rightarrow f_{*}: B_{1} \rightarrow Y_{*}$. From (1), we can fix $\lambda$ so that

$$
\int_{\partial B_{\lambda}}\left|\frac{\partial f_{\sigma_{i}}}{\partial \tau}\right|^{2} d s \rightarrow \int_{\partial B_{\lambda}}\left|\frac{\partial f_{*}}{\partial \tau}\right|^{2} d s
$$

By [GS], pages 200-201, we have

$$
0 \neq \alpha=\lim _{\sigma \rightarrow 0} \frac{\sigma \lambda E(\sigma \lambda)}{\int_{\partial B_{\sigma \lambda}(p)} d^{2}\left(f, Q_{\sigma \lambda}\right) d s}=\lim _{\sigma \rightarrow 0} \frac{\sigma \lambda E(\sigma \lambda)}{\int_{\partial B_{\sigma \lambda}(p)} d^{2}(f, f(0)) d s},
$$

which then implies

$$
\lim _{\sigma \rightarrow 0} \frac{\int_{\partial B_{\sigma \lambda}(p)} d^{2}\left(f, Q_{\sigma \lambda}\right) d s}{\int_{\partial B_{\sigma \lambda}(p)} d^{2}(f, f(0)) d s}=1 .
$$

Let $Q_{i} \in Y$ be the point so that

$$
\int_{\partial B_{\sigma_{i} \lambda}(p)} d^{2}\left(f, Q_{i}\right) d s=\inf _{Q \in Y} \int_{\partial B_{\sigma_{i} \lambda}(p)} d^{2}(f, Q) d s
$$

and $\pi_{i}: Y \rightarrow T_{Q_{i}} Y$ be a projection map into the tangent cone of $Y$ at $Q_{i}$. By the previous lemma,

$$
\int_{\partial B_{\sigma_{i} \lambda}(p)} d^{2}\left(\pi_{i} \circ f, 0\right) d s=\inf _{V \in T_{Q_{i}} Y} \int_{\partial B_{\lambda \sigma_{i}}(p)} d^{2}\left(\pi_{i} \circ f, V\right) d s .
$$

Additionally,

$$
d^{2}\left(f, Q_{i}\right)=\left|\pi_{i} \circ f\right|^{2} \text { and }\left|\frac{\partial f}{\partial \tau}\right| \geq\left|\frac{\partial\left(\pi_{i} \circ f\right)}{\partial \tau}\right|
$$


since $\pi_{i}$ is distance non-increasing. Thus, by (8) and (10),

$$
\begin{aligned}
\lim _{\sigma_{i} \rightarrow 0} \frac{\lambda^{2} \int_{\partial B_{\lambda}}\left|\frac{\partial f_{\sigma_{i}}}{\partial \tau}\right|^{2} d s}{\int_{\partial B_{\lambda}} d_{\sigma_{i}}^{2}\left(f_{\sigma_{i}}, f_{\sigma_{i}}(0)\right) d s} & =\lim _{\sigma_{i} \rightarrow 0} \frac{\left(\sigma_{i} \lambda\right)^{2} \int_{\partial B_{\sigma_{i} \lambda}}\left|\frac{\partial f}{\partial \tau}\right|^{2} d s}{\int_{\partial B_{\sigma_{i} \lambda}} d^{2}(f, f(0)) d s} \\
& =\lim _{\sigma_{i} \rightarrow 0} \frac{\left(\sigma_{i} \lambda\right)^{2} \int_{\partial B_{\sigma_{i} \lambda}}\left|\frac{\partial f}{\partial \tau}\right|^{2} d s}{\int_{\partial B_{\sigma_{i} \lambda}} d^{2}\left(f, Q_{i}\right) d s} \\
& \geq \lim _{\sigma_{i} \rightarrow 0} \frac{\left(\sigma_{i} \lambda\right)^{2} \int_{\partial B_{\sigma_{i} \lambda}}\left|\frac{\partial\left(\pi_{i} \circ f\right)}{\partial \tau}\right|^{2} d s}{\int_{\partial B_{\sigma_{i} \lambda}}\left|\pi_{i} \circ f\right|^{2} d s} .
\end{aligned}
$$

By change of coordinates $y=\sigma_{i} \lambda x,(9)$ and Corollary 15,

$$
\begin{aligned}
\frac{\left(\sigma_{i} \lambda\right)^{2} \int_{y \in \partial B_{\sigma_{i} \lambda}}\left|\frac{\partial\left(\pi_{i} \circ f\right)}{\partial \tau}(y)\right|^{2} d s}{\int_{\partial B_{\sigma_{i} \lambda}}\left|\pi_{i} \circ f(y)\right|^{2} d s} & =\frac{\int_{x \in \partial B_{1}}\left|\frac{\partial\left(\pi_{i} \circ f \circ\left(\sigma_{i} \lambda\right)\right)}{\partial \tau}(x)\right|^{2} d s}{\int_{x \in \partial B_{1}}\left|\pi_{i} \circ f \circ\left(\sigma_{i} \lambda\right)(x)\right|^{2} d s} \\
& =\frac{\int_{x \in L k(p)}\left|\frac{\partial\left(\pi_{i} \circ f \circ\left(\sigma_{i} \lambda\right)\right)}{\partial \tau}(x)\right|^{2} d s}{\int_{x \in L k(p)}\left|\pi_{i} \circ f \circ\left(\sigma_{i} \lambda\right)(x)\right|^{2} d s} \\
& \geq \lambda_{1}\left(L k(v), T_{Q_{i}} Y\right) \\
& \geq \beta(>\beta) .
\end{aligned}
$$

Therefore,

$$
\begin{aligned}
R & :=\frac{\int_{\partial B_{1}}\left|\frac{\partial f_{*}}{\partial \tau}\right|^{2} d s}{\int_{\partial B_{1}} d^{2}\left(f_{*}, f_{*}(0)\right) d s} \\
& =\frac{\lambda^{2} \int_{\partial B_{\lambda}}\left|\frac{\partial f_{*}}{\partial \tau}\right|^{2} d s}{\int_{\partial B_{\lambda}} d^{2}\left(f_{*}, f_{*}(0)\right) d s} \\
& =\lim _{\sigma_{i} \rightarrow 0} \frac{\lambda^{2} \int_{\partial B_{\lambda}}\left|\frac{\partial f_{\sigma_{i}}}{\partial \tau}\right|^{2} d s}{\int_{\partial B_{\lambda}} d_{\sigma_{i}}^{2}\left(f_{\sigma_{i}}, f_{\sigma_{i}}(0)\right) d s} \\
& \geq \beta(>\beta) .
\end{aligned}
$$

For $y \in \partial B_{1}$, the homogeneity of $f_{*}$ implies

$$
d\left(f_{*}(r y), f_{*}(0)\right)=r^{\alpha} d\left(f_{*}(y), f_{*}(0)\right),
$$

and hence

$$
E^{f_{*}}(1)=\int_{y \in \partial B_{1}} \int_{0}^{1}\left|\frac{\partial f_{*}}{\partial r}(r y)\right|^{2}+\frac{1}{r^{2}}\left|\frac{\partial f_{*}}{\partial \tau}(r y)\right|^{2} r d r d s
$$




$$
\begin{aligned}
& =\int_{y \in \partial B_{1}} \int_{0}^{1} \alpha^{2} r^{2 \alpha-1} d^{2}\left(f_{*}(y), f_{*}(0)\right)+r^{2 \alpha-1}\left|\frac{\partial f_{*}}{\partial \tau}(y)\right|^{2} d r d s \\
& =\frac{\alpha}{2} \int_{y \in \partial B_{1}} d^{2}\left(f_{*}(y), f_{*}(0)\right)+\frac{1}{2 \alpha}\left|\frac{\partial f_{*}}{\partial \tau}(y)\right|^{2} d r d s
\end{aligned}
$$

Thus,

$$
\alpha=\frac{E^{f_{*}}(1)}{I^{f_{*}}(1)}=\frac{\alpha}{2}+\frac{1}{2 \alpha} R
$$

and

$$
\alpha^{2}=R \geq \beta(>\beta)
$$

Q.E.D.

\section{The fixed point and rigidity theorems}

We can now record our main theorem.

Theorem 17 Let $X$ be an admissible, Euclidean 2-complex with weight $w$ and $Y$ an NPC space. Assume $\lambda_{1}\left(L k(p), T_{Q} Y\right) \geq 1$ for all $p \in X^{(0)}$ and all $Q \in Y$. If $f: X \rightarrow Y$ is a w-harmonic map, then $f$ is totally geodesic on each 2-simplex $F$ of $X$. If the curvature of $Y$ is strictly negative, $f$ maps each 2-simplex into a geodesic of $Y$. Furthermore, if $\lambda_{1}\left(L k(p), T_{Q} Y\right)>1$ for all $p \in X^{(0)}$ and all $Q \in Y$, then $f$ is constant.

Proof. Follows immediately from Theorem 12, Theorem 13 and Theorem 16. Q.E.D.

An important example of NPC space is the Weil-Petersson completion $\overline{\mathcal{T}}$ of Teichmüller space $\mathcal{T}$ marked genus g, n-punctured Riemann surfaces. Recently Wolpert showed that the tangent cone of $\overline{\mathcal{T}}$ at a point in the boundary is isometric to the tangent cone of $\mathbf{R}_{\geq \mathbf{0}}^{\mathbf{k}} \times \mathcal{T}^{\prime}$, where $\mathbf{R}_{>\mathbf{0}}^{\mathbf{k}}$ is the half space in $\mathbf{R}^{k}$ and $\mathcal{T}^{\prime}$ is a lower genus Teichmüller space (cf. [Wo]). We can thus deduce the following theorem:

Theorem 18 Let $X$ be a 2-dimensional admissible simplicial complex such that $\lambda_{1}(L k(p)) \geq 1$ for all $p \in X^{(0)}$. Then any w-harmonic map to the Weil-Petersson completion of Teichmüller space is totally geodesic on each simplex of $X$. Furthermore if $\lambda_{1}(L k(p))>1$ for all $p \in X^{(0)}$ then $f$ is constant. 
Proof. By the variational definition (6) and Section 4 of [DM2]

$$
\begin{aligned}
\lambda_{1}\left(\operatorname{Lk}(p), T_{Q} \overline{\mathcal{T}}\right) & =\lambda_{1}\left(\operatorname{Lk}(p), \mathbf{R}_{\geq \mathbf{0}}^{\mathrm{k}} \times \mathbf{R}^{\mathbf{l}}\right) \\
& \geq \lambda_{1}\left(\operatorname{Lk}(p), \mathbf{R}^{k+l}\right) \\
& =\lambda_{1}(\operatorname{Lk}(p), \mathbf{R}) .
\end{aligned}
$$

It follows that in this case the condition $\lambda_{1}\left(\operatorname{Lk}(p), T_{Q} \overline{\mathcal{T}}\right) \geq 1$ can be replaced by the simpler condition $\lambda_{1}(\operatorname{Lk}(p)) \geq 1$, which immediately yields the result. Q.E.D.

As noted in the introduction, it follows from [DM2] that the condition $\lambda_{1}(\operatorname{Lk}(p)) \geq 1(>1)$ is equivalent to the combinatorial condition $\lambda_{1}^{c o m b}(\operatorname{Lk}(p)) \geq 1 / 2(>1 / 2)$ in the special case when 2-simplices of $X$ are isometric to equilateral triangles. Hence Theorem 18 immediately implies the rigidity of representations of $\Gamma=\pi_{1}(X)$ into the mapping class group in the case when the complex $X$ satisfies the combinatorial condition $\lambda_{1}^{c o m b}(\operatorname{Lk}(p))>1 / 2$ for all $p \in X^{(0)}$ (cf. also [Wo]).

\section{Appendix}

Let $R=[0,1] \times[0,1] \subset \mathbf{R}^{2}$ and $f: R \rightarrow Y$ be a Lipschitz energy minimizing map with Lipschitz constant $L$ so that the pull-back inner product $\pi=\left(\pi_{i j}\right)$ is non-degenerate and smooth in $\bar{R}$. The purpose of the appendix is to show:

Theorem 19 The smooth Riemannian manifold $(R, \pi)$ is a non-positively curved surface.

Remark. Petrunin $[\mathrm{P}]$ claims a more general result. Namely, given any metric minimizing map (which includes energy minimizing maps), he states that the pull back metric (see below) defines a NPC space. Because many of the details are only sketched in his paper, we provide a complete proof here for the specific case that we need (cf. proof of Theorem 13) based on the outline in his paper.

The rest of this section is devoted to its proof of Theorem 19 .

For any spaces $\Omega_{1}$ and $\Omega_{2}$, a distance function $d$ on $\Omega_{2}$ and a Lipschitz map $g: \Omega_{1} \rightarrow\left(\Omega_{2}, d\right)$, we define two types of pull backs of $d$. The 
pull back distance function $\rho^{g}: \Omega_{1} \times \Omega_{1} \rightarrow \mathbf{R}^{+}$is

$$
\rho^{g}(z, w)=d(g(z), g(w))
$$

and the pull back metric $d^{g}: \Omega_{1} \times \Omega_{1} \rightarrow \mathbf{R}^{+}$is

$$
d^{g}(z, w)=\inf _{\gamma \in \Lambda} \operatorname{length}(g \circ \gamma)
$$

where $\Lambda$ is the set of all Lipschitz curves $\gamma:[0,1] \rightarrow R$ with $\gamma(0)=z$ and $\gamma(1)=w$. Generally, these are only pseudo-distance functions, but we will refer to them as distance functions by an abuse terminology. Clearly, we have the inequality

$$
\rho^{g}(z, w) \leq d^{g}(z, w)
$$

but, if $g\left(\Omega_{1}\right)=\Omega_{2}$ then

$$
\rho^{g}(z, w)=d^{g}(z, w)
$$

Let

$$
R(n)=\left\{z_{i j}=\left(\frac{i}{2^{n}}, \frac{j}{2^{n}}\right): i, j=0, \ldots, 2^{n}\right\},
$$

$\partial R(n)=R(n) \cap \partial R$ and $R^{\prime}(n)=R(n)-\partial R(n)$. We let $R_{i j}$ denote the box defined by $z_{i j}, z_{i j+1}, z_{i+1 j+1}$ and $z_{i+1 j}$. Two points $z, z^{\prime} \in R(n)$ are said to be adjacent if $z=\left(\frac{i}{2^{n}}, \frac{j}{2^{n}}\right)$ and $z^{\prime}=\left(\frac{i \pm 1}{2^{n}}, \frac{j}{2^{n}}\right)$ or $z^{\prime}=\left(\frac{i}{2^{n}}, \frac{j \pm 1}{2^{n}}\right)$. We will write this relationship by $z * z^{\prime}$.

Since $\pi$ is a smooth inner product, $d^{f}$ is equal to the distance function induced by $\pi$, i.e.

$$
d^{f}(z, w)=\inf _{\gamma \in \Lambda} \int_{0}^{1} \sqrt{\pi\left(\gamma^{\prime}(t), \gamma^{\prime}(t)\right)} d t .
$$

The smoothness of $\pi$ also implies that there exists $\kappa$ so that the Gaussian curvature of $R$ is less that $\kappa>0$. For $n$ sufficiently large, there exists a convex quadrilateral $\bar{Q}_{i j}$ in a sphere of constant curvature $\kappa$ with side lengths of $\bar{Q}_{i j}$ equal to those of $R_{i j}$ measured with respect to $d^{f}$. Construct a piecewise spherical space $S_{n}$ by gluing together the edges of $\bar{Q}_{i j}$ in the obvious way and let $\bar{d}_{n}$ be the natural distance function defined on $S_{n}$. Since $d^{f}$ is smooth, there exists a Lipschitz homeomorphism $\alpha_{n}:\left(R, d^{f}\right) \rightarrow\left(S_{n}, \bar{d}_{n}\right)$ which takes $R_{i j}$ diffeomorphically to $\bar{Q}_{i j}$ so that

$$
d^{\alpha_{n}}(z, w) \leq d^{f}(z, w)+O(n)|z-w|
$$


for $z, w \in R$ and where $O(n) \rightarrow 0$ as $n \rightarrow \infty$. Let $\left\{e_{1}, e_{2}\right\}$ be the standard orthonormal vectors in $R$ and $\gamma_{0}(t)=z+t \epsilon e_{i}$. Then

$$
d^{f}\left(z, z+\epsilon e_{i}\right) \leq \int_{0}^{1} \sqrt{\pi\left(\gamma_{0}^{\prime}(t), \gamma_{0}^{\prime}(t)\right)} d t \leq \epsilon \sup _{t \in[0,1]} \sqrt{\pi_{i i}\left(\gamma_{0}(t)\right)} .
$$

By the smoothness of $\pi$, it follows that

$$
\lim _{\epsilon \rightarrow 0} \frac{d^{f}\left(z, z+\epsilon e_{i}\right)^{2}}{\epsilon^{2}} \leq \pi_{i i}(z) .
$$

On the other hand, (11) implies

$$
\frac{\rho^{\alpha_{n}}\left(z, z+\epsilon e_{i}\right)^{2}}{\epsilon^{2}} \leq \frac{d^{\alpha}\left(z, z+\epsilon e_{i}\right)^{2}}{\epsilon^{2}}
$$

and hence

$$
\left|\left(\alpha_{n}\right)_{*}\left(e_{i}\right)\right|^{2} \leq \lim _{\epsilon \rightarrow 0} \frac{d^{\alpha}\left(z, z+\epsilon e_{i}\right)^{2}}{\epsilon^{2}}
$$

for a.e. $z \in R$ by Lemma 1.9.4 of [KS1]. Combining (13), (14) and (15), we obtain

$$
E^{\alpha_{n}} \leq E^{f}+O(n) .
$$

Let $\mathcal{F}_{n}$ be a set of maps $\psi: R(n) \rightarrow Y$ so that

$$
\text { (i) }\left.\psi\right|_{\partial R(n)}=\left.f\right|_{\partial R(n)}
$$

and, for $z, z^{\prime} \in R(n)$ with $z * z^{\prime}$,

$$
\text { (ii) } d\left(\psi(z), \psi\left(z^{\prime}\right)\right) \leq d\left(f(z), f\left(z^{\prime}\right)\right) .
$$

Define the step $n$ discrete energy $E_{n}: \mathcal{F}_{n} \rightarrow Y$ by setting

$$
E_{n}(\psi)=\frac{1}{2} \sum_{z \in R^{\prime}(n)} \sum_{z^{\prime} * z} d^{2}\left(\psi(z), \psi\left(z^{\prime}\right)\right) .
$$

Lemma 20 There exists $\psi_{n} \in \mathcal{F}_{n}$ so that $E_{n}\left(\psi_{n}\right)=e_{n}:=\inf _{\psi} E_{n}(\psi)$ where inf is taken over all $\psi \in \mathcal{F}_{n}$.

Proof. Since $\left.f\right|_{R(n)} \in \mathcal{F}_{n}, \mathcal{F}_{n}$ is non-empty. Let $\psi^{i} \in \mathcal{F}_{n}$ so that $E_{n}\left(\psi^{i}\right) \rightarrow e_{n}$. If $\psi^{i j} \in \mathcal{F}_{n}$ is defined so that $\psi^{i j}(z)$ is the midpoint on the geodesic between $\psi^{i}(z)$ and $\psi^{j}(z)$, then

$$
\begin{aligned}
& d\left(\psi^{i j}(z), \psi^{i j}(w)\right) \\
& \leq \quad \frac{1}{2} d\left(\psi^{i}(z), \psi^{i}(w)\right)+\frac{1}{2} d\left(\psi^{j}(z), \psi^{j}(w)\right) \\
& \quad-\frac{1}{4}\left(d\left(\psi^{i}(z), \psi^{j}(z)\right)-d\left(\psi^{i}(w), \psi^{j}(w)\right)\right)^{2}
\end{aligned}
$$


and hence

$e_{n} \leq E_{n}^{\psi^{i j}} \leq \frac{1}{2} E_{n}^{\psi^{i}}+\frac{1}{2} E_{n}^{\psi^{j}}-\frac{1}{4} \sum_{z \in R^{\prime}(n)}\left(d\left(\psi^{i}(z), \psi^{j}(z)\right)-d\left(\psi^{i}(z), \psi^{j}(w)\right)\right)^{2}$

Thus,

$$
\lim _{i, j \rightarrow \infty} \sum_{z \in R^{\prime}(x)}\left(d\left(\psi^{i}(z), \psi^{j}(z)\right)-d\left(\psi^{i}(z), \psi^{j}(w)\right)\right)^{2}=0
$$

which says that $\left\{\psi^{i}(z)\right\}$ is a Cauchy sequence for each $z \in R^{\prime}(n)$ and hence $\psi^{i}(z)$ converge to $\psi_{n}(z)$ and $E_{n}\left(\psi_{n}\right)=e_{n}$ by the continuity of the distance function. Q.E.D.

Since $Y$ is an NPC space, there exists a convex quadrilateral $Q_{i j}$ in the Euclidean plane with side lengths of $R_{i j}$ measured with respect to $\rho^{\psi_{n}}$. We denote the vertices of $Q_{i j}$ corresponding to the vertices $z_{i j}, z_{i+1 j}, z_{i+1 j+1}, z_{i j+1}$ by $q_{i j}, q_{i+1 j}, q_{i+1 j+1}, q_{i j+1}$. Construct a piecewise linear space $L_{n}$ by gluing together the edges of $Q_{i j}$ in the obvious way and let $d_{n}$ be the natural distance function defined on $L_{n}$. By condition (ii) and the fact that $S_{n}$ is piecewise spherical (of constant curvature $\kappa$ ) and $L_{n}$ is piecewise Euclidean, there exists a homeomorphism $\beta_{n}: S_{n} \rightarrow\left(L_{n}, d_{n}\right)$ which takes $\bar{Q}_{i j}$ diffeomorphically to $Q_{i j}$ so that

$$
d^{\beta_{n}}(p, q) \leq \bar{d}_{n}(p, q)
$$

for $p, q \in S_{n}$. Setting $p=\alpha_{n}(z)$ and $q=\alpha_{n}(w)$, this implies that

$$
d^{\beta_{n} \circ \alpha_{n}}(z, w) \leq d^{f}(z, w)+O(n)|z-w|
$$

for $z, w \in R$ and

$$
E^{\beta_{n} \circ \alpha_{n}} \leq E^{f}+O(n)
$$

Connect the ordered points

$$
\psi_{n}\left(q_{i j}\right), \psi_{n}\left(q_{i j+1}\right), \psi_{n}\left(q_{i+1 j}\right) \text { and } \psi_{n}\left(q_{i+1 j+1}\right)
$$

by geodesics, except when the two consecutive points lie on the boundary of $L_{n}$ in which case we use the corresponding boundary value of $f$ to connect them. The resulting quadrilateral in $Y$ will be denoted by $Q_{i j}$. (Note that this modification from geodesic lines to the boundary value of $f$ becomes irrelevant as $n \rightarrow \infty$ in the sense the modified version or the unmodified version become uniformly close as $n \rightarrow \infty$.) From 
these geodesic quadrilaterals, we construct a "ruled surface" (in the sense of Alexandrov [A]). This is constructed as follows. First, choose a pair of opposite edges in $Q_{i j}$ and give a constant speed parametrization $\gamma, \sigma:[0,1] \rightarrow Y$ of these edges so that $\gamma(0)$ and $\sigma(0)$ lie on one side of the quadrilateral. Second, connect $\gamma(t)$ and $\sigma(t)$ by a geodesic for each $t \in[0,1]$. We call this surface $\mathcal{Q}_{i j}$. We define $\mathcal{R}_{n}$ as an abstract space made up of disjoint union of $\mathcal{Q}_{i j}$ with the identification along the adjacent boundaries. Note that $\mathcal{R}_{n}$ is similar to the piecewise linear space $L_{n}$ but with linear pieces replaced by ruled surfaces in $Y$.

Lemma 21 The metric space $\mathcal{R}_{n}$ is $N P C$.

Proof. By the result of Alexandrov, ruled surfaces in NPC spaces are NPC (cf. [A]). Thus, $\mathcal{R}$ is a piecewise NPC surface. Therefore, we need only to check that the total angle at the vertices, i.e $p=\psi_{n}(z)$ for $z \in R^{\prime}(n)$ where four ruled sufaces meet, are $\geq 2 \pi$. Let geodesics $l_{1}, l_{2}, l_{3}$ and $l_{4}$ be the interfaces of the four ruled surfaces meeting at $p=\psi_{n}(z)$ for some $z \in R^{\prime}(n)$. Denote the other endpoint of $l_{i}$ by $\psi_{n}\left(z_{i}\right)$ for $z_{i} * z$.

Since the distance measured along the surface is always greater or equal to the distance measured in the ambient space, we need only check that the sum of the angle between $l_{i}, l_{i+1}(i=1,2,3)$ and $l_{4}, l_{1}$ measured in terms of the distance function $d$ on $Y$ is $\geq 2 \pi$. So suppose not. Let $\left[W_{i}, t_{i}\right]$ be the endpoint of the geodesic $\pi\left(l_{i}\right)$ emanating from the origin where $\pi: Y \rightarrow T_{p} Y$ is the projection map to the tangent cone at $\psi_{n}(z)=p$. Since $\pi$ is a non-expanding map, $<W_{i}, W_{i+1}>\leq \angle\left(l_{i}, l_{i+1}\right)$. Since $W_{1}, \ldots, W_{4}$ are points in the space of directions, $S_{p} Y$, which is a CAT(1) space, there exists a convex quadrilateral $\bar{Q}$ in $S^{2}$ with vertices $\bar{W}_{1}, \ldots, \bar{W}_{4}$ preserving distances of $W_{1}, \ldots, W_{4}$ and a non-expanding map $r: \bar{Q} \rightarrow S_{p} Y$. Since $\bar{Q}$ has length $<2 \pi$, we can assume $\bar{Q}$ is compactly contained in the upper hemisphere. Thus, if $N$ is the north pole of $S^{2}$, then $<\bar{W}_{i}, N><\frac{\pi}{2}$ which then implies $<W_{i}, r(N)><\frac{\pi}{2}$. Hence

$$
\begin{aligned}
\frac{d}{d \tau} d_{T_{P} Y}^{2}\left([r(N), \tau],\left[W_{i}, t_{i}\right]\right) & =\frac{d}{d \tau}\left(\tau^{2}+t_{i}^{2}-2 \tau t_{i} \cos <W_{i}, r(N)>\right) \\
& =2 \tau-2 t_{i} \cos <W_{i}, r(N)><0
\end{aligned}
$$

for small $\tau>0$ which then implies

$$
\tau \mapsto \sum_{i=1}^{4} d_{T_{P} Y}^{2}\left([r(N), \tau], \pi \circ \psi_{n}\left(z_{i}\right)\right)
$$


is a decreasing function for small $\tau>0$. On the other hand, $\psi_{n}$ is a step $n$ energy minimizing map, which implies that $0 \in T_{f(z)} Y$ is the center of mass of $\pi \circ \psi_{n}\left(z_{1}\right), \pi \circ \psi_{n}\left(z_{2}\right), \pi \circ \psi_{n}\left(z_{3}\right)$ and $\pi \circ \psi_{n}\left(z_{4}\right)$ (cf. [IN]). This contradiction implies

$$
\angle\left(l_{1}, l_{2}\right)+\angle\left(l_{2}, l_{3}\right)+\angle\left(l_{3}, l_{4}\right)+\angle\left(l_{4}, l_{1}\right) \geq 2 \pi \quad(i \bmod 4)
$$

which proves our assertion. Q.E.D.

By a theorem of Reshetnyak [R] (see also [KS1], Theorems 2.1.1 and 2.1.2), there exists a non-expanding map $\gamma_{n}: L_{n} \rightarrow \mathcal{R}_{n}$ with $\gamma_{n}\left(q_{i j}\right)=$ $\psi_{n}\left(z_{i j}\right), \gamma_{n}\left(q_{i j+1}\right)=\psi_{n}\left(z_{i j+1}\right), \gamma_{n}\left(q_{i+1 j}\right)=\psi_{n}\left(z_{i+1 j}\right)$ and $\gamma_{n}\left(q_{i+1 j+1}\right)=$ $\psi_{n}\left(z_{i+1 j+1}\right)$. Thus,

$$
d^{\gamma_{n}}(r, s) \leq d_{n}(r, s)
$$

for $r, s \in L_{n}$. We define

$$
f_{n}: R \rightarrow Y, \quad \text { by } \quad f_{n}=\iota_{n} \circ \gamma_{n} \circ \beta_{n} \circ \alpha_{n},
$$

where $\iota_{n}: \mathcal{R}_{n} \rightarrow Y$ is a map which embedds each ruled surface $\mathcal{Q}_{i j}$ into $Y$ in the obvious way. Set $r=\beta_{n} \circ \alpha_{n}(z)$ and $s=\beta_{n} \circ \alpha_{n}(w)$, we see that

$$
d^{f_{n}}(z, w) \leq d^{f}(z, w)+O(n)|z-w| .
$$

and

$$
E^{f_{n}} \leq E^{f}+O(n) .
$$

Therefore, $f_{n}$ is a minimizing sequence converging uniformly in the pull back sense to $f$ by Theorem 3.11 of [KS2].

We set $\bar{f}_{n}=\gamma_{n} \circ \beta_{n} \circ \alpha_{n}$, i.e. $\bar{f}_{n}: R \rightarrow \mathcal{R}_{n}$ is $f_{n}$ viewed as a map with target $\mathcal{R}_{n}$. Since $\bar{f}_{n}(R)=\mathcal{R}_{n}$, we have

$$
d^{\bar{f}_{n}}=\rho^{\bar{f}_{n}} .
$$

By construction, $\bar{f}_{n}: R \rightarrow \mathcal{R}_{n}$ is a uniformly Lipschitz sequence of maps into NPC spaces. Proposition 3.7 of [KS2] implies that there exists a subsequence (denoted again $\bar{f}_{n}$ by an abuse of notation) so that it converges locally uniformly in the pull back sense to a map $\bar{f}_{*}: R \rightarrow \mathcal{R}_{*}$. In particular, this means that $d^{\bar{f}_{n}}=\rho^{\bar{f}_{n}}$ converges uniformly to $\rho^{\bar{f}_{*}}$ which equals $d^{\bar{f}_{*}}$ by definition of Korevaar-Schoen limit (cf. [KS2]). Since $\iota_{n}$ is a piecewise isometry, we see that $d^{f_{n}}=d^{\bar{f}_{n}}$ and $\pi^{f_{n}}$ and $\pi^{\bar{f}_{n}}$ (the pull back inner products of $f_{n}$ and $\bar{f}_{n}$ respectively) agree a.e.. Since $\pi^{f_{n}}$ converges to $\pi^{f}$ and $\pi^{\bar{f}_{n}}$ converges to $\pi^{\bar{f}_{*}}$, we see 
that $\pi^{f}=\pi^{f_{n}}$ which immediately implies that $d^{f}=d^{\bar{f}_{*}}$ by smoothness. In conclusion, we have shown that $d^{f_{n}}=d^{\bar{f}_{n}}$ converges uniformly to $d^{f}$.

Since $\mathcal{R}_{n}$ is an NPC space and $\bar{f}_{n}(R)=\mathcal{R}_{n}, d^{\bar{f}_{n}}$ defines a NPC distance function on $R$ (after identifications of points of zero $d^{\bar{f}_{n}}$ distance). Thus, the uniform convergence of $d^{\bar{f}_{n}}$ to $d^{f}$ implies that $d^{f}$ defines an NPC space. This shows that $(R, \pi)$ is a smooth manifold of non-positive curvature.

\section{References}

[A] A.D. Alexandrov. Ruled surfaces in metric spaces. Vestnik LGU Ser. Mat., Mech., Astr. vyp. 1, (1957) 5-26.

[BH] M. Bridson and A. Haefliger. Metric spaces of non-positive curvature. Springer, 1991.

[Ch] J. Chen. On energy minimizing mappings between and into singular spaces. Duke Math. J. 79 (1995) 77-99.

[DM1] G. Daskalopoulos and C. Mese. Harmonic maps from a 2complex. Comm. Anal. Geom. 14 (2006) 497-549.

[DM2] G. Daskalopoulos and C. Mese. Harmonic maps from a simplicial complex and geometric rigidity. To appear Jour. of Diff. Geo.

[EF] J. Eells and B. Fuglede. Harmonic maps between Riemannian polyhedra. Cambridge Tracts in Mathematics 142, Cambridge University Press, Cambridge 2001.

[GS] M. Gromov and R. Schoen. Harmonic maps into singular spaces and p-adic superrigidity for lattices in groups of rank one. Publ. Math. IHES 76 (1992) 165-246.

[IN] H. Izeki and S. Nayatani. Combinatorial harmonic maps and discrete-group actions on Hadamard spaces. Geom. Dedicata 114 (2005) 147-188.

[KS1] N. Korevaar and R. Schoen. Sobolev spaces and harmonic maps into metric space targets. Comm. Anal. Geom. 1 (1993) 561-659.

[KS2] N. Korevaar and R. Schoen. Global existence theorem for harmonic maps to non-locally compact spaces. Comm. Anal. Geom. 5 (1997), 333-387. 
[P] A. Petrunin. Metric minimizing surfaces. Electron. Res. Announc. Amer. Math. Soc 5 (1999) 47-54.

[R] Y.G. Reshetnyak. Non-expansive maps in a space of curvature no greater than K. Siberian Math. J. 9 (1968) 683-689.

[W1] M.-T. Wang. A fixed point theorem of discrete group actions on Riemannian manifolds. J. Diff. Geom. 50 (1998) 249-267.

[W2] M.-T. Wang. Generalized harmonic maps and representations of discrete groups. Comm. Anal. Geom. 8 (2000) 545-563.

[Wo] S. Wolpert. Behaviour of geodesic-length functions on Teichmüller space. Preprint 2007. 